\title{
Proceedings
}

\section{Testing a Best-Fit Hydrodynamical Model Using PCA ${ }^{+}$}

\author{
Tiago Nunes da Silva ${ }^{1,3, *}$, David Dobrigkeit Chinellato ${ }^{1}$, Rafael Derradi de Souza ${ }^{1}$, \\ Maurício Hippert ${ }^{2}$, Matthew Luzum ${ }^{2} \mathbb{D}$, Jorge Noronha ${ }^{2}$ and Jun Takahashi ${ }^{1}$ \\ 1 Instituto de Física Gleb Wataghin, Universidade Estadual de Campinas, R. Sérgio Buarque de Holanda, 777, \\ Campinas 13083-859, Brazil; daviddc@unicamp.br (D.D.C.); rderradi@ifi.unicamp.br (R.D.d.S.); \\ jun@ifi.unicamp.br (J.T.) \\ 2 Instituto de Física, Universidade de São Paulo, R. do Matão, 1371, São Paulo 05508-090, Brazil; \\ hippert@if.usp.br (M.H.); mluzum@usp.br (M.L.); noronha@if.usp.br (J.N.) \\ 3 Present Address: Centro de Ciências Físicas e Matemáticas, Universidade Federal de Santa Catarina, Campus \\ Universitário Reitor João David Ferreira Lima, Florianópolis 88040-900, Brazil \\ * Correspondence: t.j.nunes@ufsc.br \\ + Presented at Hot Quarks 2018-Workshop for young scientists on the physics of ultrarelativistic \\ nucleus-nucleus collisions, Texel, The Netherlands, 7-14 September 2018
}

Published: 10 April 2019

check for updates

\begin{abstract}
Recently, a comprehensive Bayesian analysis was performed to simultaneously extract the values of a number of hydrodynamic parameters necessary for compatibility with a limited set of experimental data from the LHC. In this work, this best-fit model is tested against newly measured experimental flow results not included in the original work, namely the principal components of the two-particle correlation matrix in transverse momentum. The results from simulations show a good numerical agreement with data obtained by the CMS Collaboration.
\end{abstract}

Keywords: heavy-ion collisions; quark-gluon plasma; relativistic fluid dynamics; principal component analysis

\section{Introduction}

Relativistic heavy-ion collision (HIC) experiments have proven to be an important tool in exploring the fundamental nature of strongly interacting matter under extreme conditions. The standard picture of the processes involved in such collisions is that after a short period of time following the collision of the original nuclei, the evolution of the resulting system can be described by relativistic viscous hydrodynamics. In fact, simulations of heavy-ion collisions based on hydrodynamical evolution are able to describe several observables from experimental data with great accuracy [1]. The hydrodynamical evolution can be complemented by a subsequent simulation of hadronic cascade models in order to describe the evolution of the gas of hadrons formed after the fluid cools down and the particles hadronize. This combination is usually referred to as a hybrid model [2].

Recently, Bernhard et al. used a hybrid model [3,4] consisting of the TRENTo model [5] for the generation of initial conditions, the VISH $2+1$ code for hydrodynamical evolution, and the UrQMD transport model [6,7] for the evolution of the hadron gas phase. Through a Bayesian analysis the authors have obtained the optimal a posteriori values for a series of parameters required by the model. In this work we utilize a similar setup and test the validity of the model with these parameters for a set of new observables not included in the original Bayesian analysis, namely results from a principal component analysis (PCA) of the two-particle correlation matrix in transverse momentum [8]. 


\section{Materials and Methods}

The hybrid model for HIC simulations devised in this work is comprised of the following ingredients:

- TRENTo, a parametric wounded nucleon model for generation of initial conditions for hydrodynamics in HIC [5];

- $\quad$ MUSIC, an Eulerian 3D+1 relativistic second-order viscous hydrodynamics code for event by event HIC simulations $[9,10]$;

- UrQMD, a transport model for the evolution of the hadron gas $[6,7]$.

Parameter values for the simulation chain were obtained from the work by Bernhard et al. [4]. We have chosen not to include in our simulation chain a period of free streaming between the initial condition generation and the beginning of the hydrodynamical evolution, i.e., we utilize the output from TRENTo as the initial distribution of entropy density for hydrodynamics. Because of that, the overall normalization constant for TRENTo had to be recalculated, which was done by matching the charged-particle multiplicity density to experimental data from the ALICE Collaboration [11,12]. It is possible that removing the free streaming period and setting an earlier initial time for hydrodynamics may impact some observables. We plan to address this possibility in a future work.

We have also developed a ROOT-based C++ class for storing the resulting final configuration of particles from each event, called HadrEx.

The Q-cumulants of the event sample are built in bins of transverse momentum following the definition by Bhalerao et al. [8]

$$
Q_{n}\left(p_{a}\right) \equiv \frac{1}{2 \pi \Delta p_{t} \Delta \eta} \sum_{j=1}^{M\left(p_{a}\right)} \exp \left(i n \varphi_{j}\right)
$$

where $p_{a}$ refers to bin $a$ in transverse momentum, and $M\left(p_{a}\right), \Delta p_{t}$, and $\Delta \eta$ refer, respectively, to the bin multiplicity and the bin ranges in transverse momentum and pseudo-rapidity.

The pair distribution is then obtained through the formula

$$
V_{n \Delta}\left(p_{a}, p_{b}\right) \equiv\left\langle Q_{n}\left(p_{a}\right) Q_{n}^{*}\left(p_{b}\right)\right\rangle-\frac{\left\langle M\left(p_{a}\right)\right\rangle \delta_{p_{a}, p_{b}}}{\left(2 \pi \Delta p_{t} \Delta \eta\right)^{2}}
$$

where the first term is the two-particle correlation matrix and the second term removes self-correlations.

The principal components are calculated by diagonalizing the matrix $V_{n \Delta}\left(p_{a}, p_{b}\right)$ and identifying the results with the PCA approximation

$$
V_{n \Delta}\left(p_{a}, p_{b}\right)=\sum_{\alpha} \lambda^{(\alpha)} \psi^{(\alpha)}\left(p_{a}\right) \psi^{(\alpha) *}\left(p_{b}\right) \approx \sum_{\alpha=1}^{k} V_{n}^{(\alpha)}\left(p_{a}\right) V_{n}^{(\alpha) *}\left(p_{b}\right),
$$

so that

$$
V_{n}^{(\alpha)}(p) \equiv \sqrt{\lambda^{\alpha}} \psi^{(\alpha)}(p) \text { and } v_{n}^{(\alpha)}(p) \equiv \frac{V_{n}^{(\alpha)}(p)}{\left\langle V_{0}(p)\right\rangle}
$$

express the principal component $\alpha$ of the $n$-th harmonic of the anisotropic flow $V_{n}$ in terms of the eigenvalues $\lambda^{(\alpha)}$ and their associated eigenvectors $\psi^{(\alpha)}$, with the eigenvalues ordered from largest to smallest. The normalization in the second equation allows for a direct comparison with the usual measurement of the differential flow.

\section{Results}

We have performed event by event simulations of collisions between $\mathrm{Pb}$ nuclei at center of mass energies $\sqrt{s_{N N}}=2.76 \mathrm{TeV}$ and $\sqrt{s_{N N}}=5.02 \mathrm{TeV}$. A sample of one million initial conditions was 
generated for centrality calibration, which was based on total entropy. Because of the strong correlation between entropy and final charged particle multiplicity, this definition is essentially equivalent to what is done experimentally. The resulting entropy distribution allows us to classify the centrality of a given event.

The resulting charged particle multiplicity distribution as a function of event centrality is presented in Figure 1. The results from simulations are compared to data from the ALICE Collaboration [11,12]. Up to the centrality bin corresponding to $50 \%$ to $60 \%$, simulation results agree with experimental data to $10 \%$ accuracy.

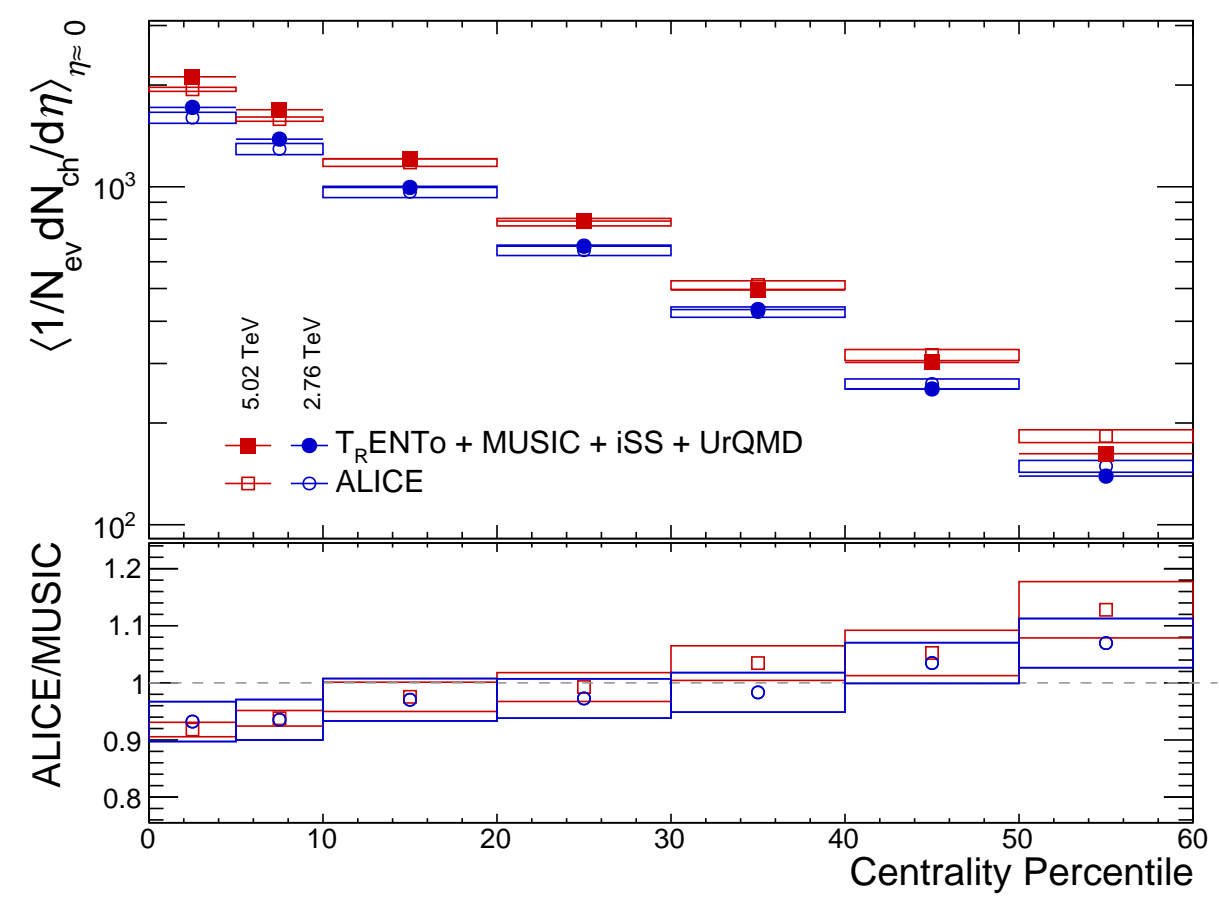

Figure 1. Charged particle multiplicity from simulations of $\mathrm{Pb}-\mathrm{Pb}$ collisions at $\sqrt{s_{N N}}=2.76 \mathrm{TeV}$ and $\sqrt{s_{N N}}=5.02 \mathrm{TeV}$ as a function of centrality. Results are compared to data from the ALICE Collaboration [11,12].

The main result of this work is the first calculation of PCA of the two-particle correlation matrix in transverse momentum using a hybrid model and realistic hydrodynamical initial conditions. A subset of these results is shown in Figure 2 (full results will be reported in a forthcoming work). 


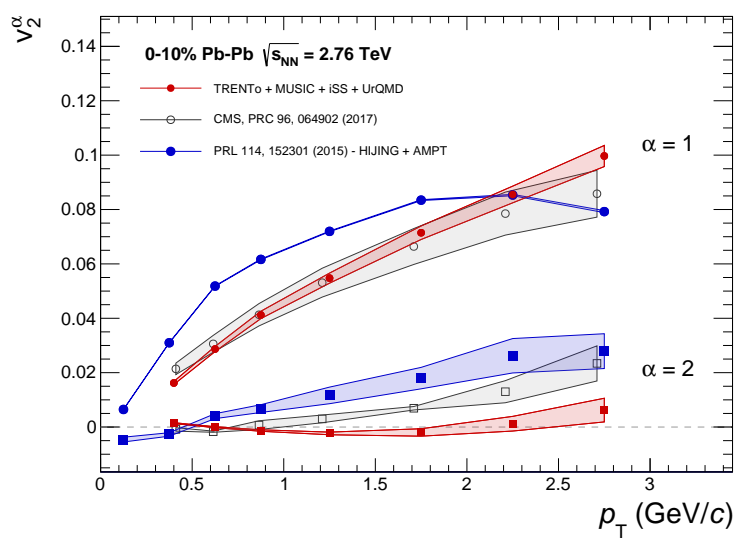

(a)

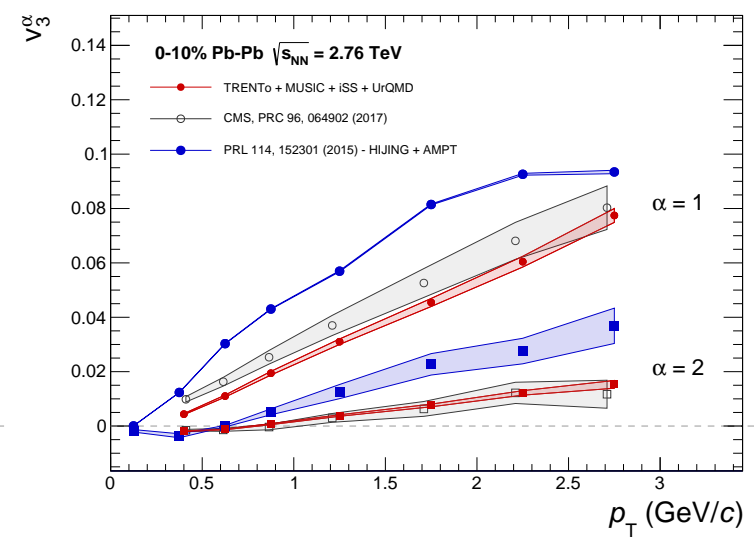

(b)

Figure 2. Principal components of the two-particle correlation matrix for $\mathrm{Pb}-\mathrm{Pb}$ collisions at center of mass energy $\sqrt{s_{N N}}=2.76 \mathrm{TeV}$ and $0 \%$ to $10 \%$ centrality for $n=2(2(\mathrm{a}))$ and for $n=3(2(\mathrm{~b}))$. Results from this work (red) are compared to the original PCA work [8] (blue) and to data from the CMS Collaboration [13] (gray).

\section{Discussion}

The principal components of the two-particle correlation matrix are an interesting observable to study: by considering the full covariance matrix, including the off-diagonal terms correlating particles lying in different bins of transverse momentum, they contain more information about the anisotropic flow than the usual measurements of the differential flow coefficients via two-particle correlations, which only correlate particles in the same transverse momentum bin. The $p_{T}$-dependent event-by-event fluctuations break factorization of the pair distribution

$$
\left\langle\frac{d N_{\text {pairs }}}{d \vec{p}_{a} d \vec{p}_{b}}\right\rangle=\left\langle\frac{d N}{d \vec{p}_{a}} \frac{d N}{d \vec{p}_{b}}\right\rangle
$$

into a product of single-particle probability distributions (as expected in pure hydrodynamics simulations without event-by-event fluctuations). The subleading components of the PCA measure the size of these fluctuations [8].

It is noteworthy that, even though these observables were not included in the original Bayesian analysis, in general we find a good agreement between results from simulations and experimental results from the CMS Collaboration [13]. While there is agreement within uncertainties in all centralities for $\alpha=1$, this is not so for $\alpha=2$, in which case the description is not adequate for central events but improves for less central events and $p_{T}<2.0 \mathrm{GeV}$. Results for other centrality ranges will be presented in future works. We will also present an extended analysis of further observables (such as symmetric cumulants) and extend this simulation framework to consider lower energies and small systems.

Author Contributions: Conceptualization, all; methodology, T.N.d.S., M.H. and M.L.; software, T.N.d.S., D.C., R.D.d.S. and M.L.; validation, T.N.d.S., D.C., R.D.d.S. and M.H.; formal analysis, T.N.d.S. and D.C.; investigation, all; resources, D.C., M.L. and J.T.; data curation, T.N.d.S. and M.L.; writing-original draft preparation, T.N.d.S.; writing-review and editing, all; visualization, T.N.d.S. and D.C.; supervision, D.C., M.L., J.N. and J.T.; project administration, J.T.; funding acquisition, J.T.

Funding: This research was funded by FAPESP grants number 2014/09167-8 (R.D.d.S.), 2016/13803-2 (D.D.C.), 2016/24029-6 (M.L.), 2017/05685-2 (all), 2018/01245-0 (T.N.d.S.) and 2018/07833-1(M.H.). D.D.C., M.L., J.N. and J.T. thank CNPq for financial support.

Acknowledgments: We would like to acknowledge computing time provided by the Research Computing Support Group at Rice University through agreement with the University of São Paulo.

Conflicts of Interest: The authors declare no conflict of interest. 


\section{References}

1. Heinz, U.; Snellings, R. Collective flow and viscosity in relativistic heavy-ion collisions. Ann. Rev. Nucl. Part. Sci. 2013, 63, 123-151.

2. Petersen, H.; Steinheimer, J.; Burau, G.; Bleicher, M.; Stöcker, H. Fully integrated transport approach to heavy ion reactions with an intermediate hydrodynamic stage. Phys. Rev. 2008, C78, 044901.

3. Bernhard, J.E.; Moreland, J.S.; Bass, S.A.; Liu, J.; Heinz, U. Applying Bayesian parameter estimation to relativistic heavy-ion collisions: simultaneous characterization of the initial state and quark-gluon plasma medium. Phys. Rev. 2016, C94, 024907.

4. Bernhard, J.E. Bayesian Parameter Estimation for Relativistic Heavy-Ion Collisions. Ph.D. Thesis, Duke University, Durham, The United States , 19 April 2018.

5. Moreland, J.S.; Bernhard, J.E.; Bass, S.A. Alternative ansatz to wounded nucleon and binary collision scaling in high-energy nuclear collisions Phys. Rev. 2015, C92, 011901.

6. Bass, S.A.; Belkacem, M.; Bleicher, M.; Brandstetter, M.; Bravina, L.; Ernst, C.; Gerland, L.; Hofmann, M.; Hofmann, S.; Konopka, J.; et al. Microscopic Models for Ultrarelativistic Heavy Ion Collisions. Prog. Part. Nucl. Phys. 1998, 41, 255-369.

7. Bleicher, M.; Zabrodin, E.; Spieles, C.; Bass, S.A.; Ernst, C.; Soff, S.; Bravina, L.; Belkacem, M.; Weber, H.; Stöcker, H.; et al. Relativistic Hadron-Hadron Collisions in the Ultra-Relativistic Quantum Molecular Dynamics Model (UrQMD). J. Phys. 1999, G25, 1859-1896.

8. Bhalerao, Rajeev S. and Ollitrault, Jean-Yves and Pal, Subrata and Teaney, Derek. Principal Component Analysis of Event-by-Event Fluctuations. Phys. Rev. Lett. 2015, 114, 152301.

9. Schenke, B.; Jeon, S.; Gale, C. (3+1)D hydrodynamic simulation of relativistic heavy-ion collisions. Phys. Rev. 2010, C82, 014903.

10. Schenke, B.; Jeon, S.; Gale, C. Higher flow harmonics from (3+1)D event-by-event viscous hydrodynamics Phys. Rev. 2012, C85, 024901.

11. Aamodt K., Abrahantes Quintana A., Adamova D., Adare A., Aggarwal M., Aglieri Rinella G., Agocs A.G., Aguilar Salazar S., Ahammed Z., Ahmad N., Ahmad Masoodi A., et al (ALICE Collaboration). Centrality Dependence of the Charged-Particle Multiplicity Density at Midrapidity in Pb-Pb Collisions at $\sqrt{s_{N N}}=2.76$ TeV. Phys. Rev. Lett. 2011, 106, 032301.

12. Adam J. , Adamova D., Aggarwal M.M., Aglieri Rinella G., Agnello M., Agrawal N., Ahammed Z., Ahmad S., Ahn S.U., Aiola S., Akindinov A., et al (ALICE Collaboration). Anisotropic Flow of Charged Particles in $\mathrm{Pb}-\mathrm{Pb}$ Collisions at $\sqrt{s_{N N}}=5.02 \mathrm{TeV}$. Phys. Rev. Lett. 2016, 116, 222302.

13. Sirunyan A.M., Tumasyan A., Adam W., Ambrogi F., Asilar E., Bergauer T., Brandstetter J., Brondolin E., Dragicevic M., Erö J., Flechl M., et al (CMS Collaboration). Principal-component analysis of two-particle azimuthal correlations in $\mathrm{PbPb}$ and $p \mathrm{~Pb}$ collisions at CMS. Phys. Rev. 2017, C96, 064902.

(C) 2019 by the authors. Licensee MDPI, Basel, Switzerland. This article is an open access article distributed under the terms and conditions of the Creative Commons Attribution (CC BY) license (http://creativecommons.org/licenses/by/4.0/). 